\title{
Node Mobility Impact on Zone Routing Protocol
}

\author{
Rakhi Purohit \\ Research Scholar \\ Department of Computer Science \& Engineering \\ Suresh Gyan Vihar University, Jaipur
}

\author{
Bright Keswani, Ph.D \\ Associate Professor \& Head \\ Department of Computer Application \\ Suresh Gyan Vihar University, Jaipur
}

\begin{abstract}
Zone Routing Protocol has designed for wireless mobile ad hoc networks. MANET is a network of geographically randomly distributed nodes. These network nodes can perform transaction of data packets using wireless links and they don't need any fixed infrastructure. In wireless mobile ad hoc network node perform task of host and router both. Because of the nodes have mobility feature so the environment of network is very much dynamic in nature, hence nodes in network are mobile in nature, they can move randomly so they can affect the topology of network and made frequent change in it in randomize mode of time slice. This paper consists of node mobility impact on zone routing protocol. This study is performed by simulation of network which has been conducted in famous network simulator NS2.
\end{abstract}

\section{General Terms}

ZRP, Routing, Simulation, NS2, Wireless Ad Hoc

\section{Keywords}

MANET, ZRP, Routing Protocol, Network Simulation, NS2, Wireless Ad Hoc Network.

\section{INTRODUCTION}

Zone Routing Protocol is a combination of two types of routing techniques reactive and proactive into one routing technique known as hybrid routing protocol [4]. ZRP minimize the control overhead of proactive routing and decrease the latency due to routing search in reactive routing protocols. The ZRP protocol makes a zone which surrounds each node consisting of its neighbor node. ZRP is reform by two supporting protocols, first proactive routing Intra-zone and second reactive routing protocol Inter-zone [13]. The IERP is used to communicate between nodes of different routing zones. In this protocol due to reactive routing technique the route discovery process performs on demand of route. Route searching process is slower, but here also the delay can be reducing by Border cast Resolution Protocol [14].

\section{ZRP OPERATIONS}

ZRP routing protocol is implemented through a separate Neighbor Discovery Protocol (NDP) using neighbor discovery. ZRP protocol specifically performs broadcasting of beacons messages in regular time durations. There after receiver node will receive this beacon message which can be used to show the status of a connection to the neighbor node which sent this message. This neighbor discovery information can be utilized for the basis for the Intra-zone Routing Protocol [15]. The IARP technique can perform by well known proactive and link state routing protocols which can provide a complete view of connectivity in network. ZRP framework route discovery process is somehow different from the well known broadcast based route searching technique. It is perform through a service which is used in the message distribution. It is also known as the Border-cast Resolution
Protocol. When he BRP protocol available then, the processing of IERP is work similar to standard route discovery protocol [15]. The IERP protocol generates a new process for route discovery when there is no any route is present in current status from source node to the destination node to sent data packets in network.

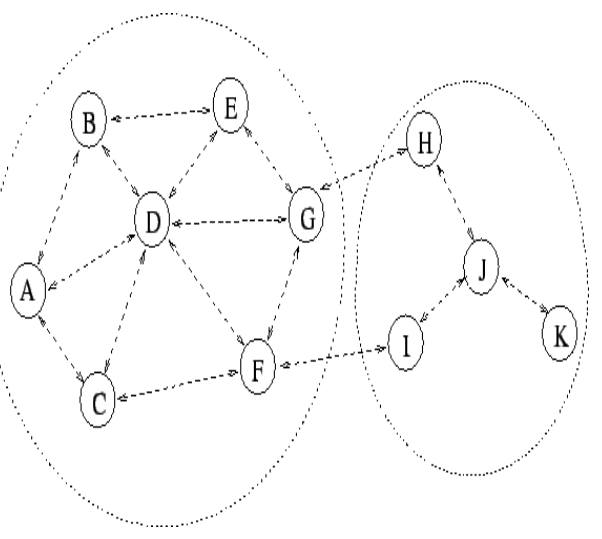

Fig. 1. ZRP Protocol Operations

\section{ARCHITECTURE}

There are two types of neighbors are there in every zone. It is either peripheral or interior nodes. ZRP consist of two types of routing proactive in the zone and reactive out of zone also known as IARP (Intra zone) and IERP (Inter zone)[13]. ZRP is using new concept known as border casting when it have to search a node outside zone so it send query request to all node present at the border of the zone so this technique is known as border casting it is perform by IARP and with help of BRP Protocol. ZRP utilize NDP to search new neighbor in network. This NDP is responsible to update routing table frequently [15]. The ZRP protocol is works on the idea of zone in network area which is consist of some nodes that come in specified zone radius of ZRP. Each node has a zone surrounding it with a particular zone radius, so many times various zones overlap one another [14]. The zone radius in ZRP is not measured by any physical distance but is it measured by hop count around center node.

\section{RELATED WORK}

A routing protocol of ad hoc networks is a good research topic and it is currently an open area. So many routing protocols proposed by the standard network communities are till now have no agreement on a solution provision due to relevance of wireless or wired networks. The researcher Asma Ahmed [3] proposed discussion on various routing protocols and also comparison between routing protocols also presents the all verity of MANET protocols and prepare a comparative output of the properties of all type of protocol. The researcher Md. Anisur Rahman [6] shows that working differentials can test using changing network load and node mobility also network size. The researcher Samyak Shah [7] checked by comparing 
the capability of protocols DSDV protocol, AODV protocol and DSR protocols of MANET with the use of network simulations. The researcher Furqan Haq [9] proposed a comparing of emulated test bed and result of simulation of NS2 simulator and GloMoSim. They use OLSR protocol. The researcher Kapang Lego [12] has done evaluation using simulation for comparison of various performance matrices on various scenarios for MANET. Some of these papers discussed the actual simulation results and also changing of found results between various network simulators. On the base of the output of the all research, there is a need for deep and correct planned network simulation and emulation test experiments has been think to required. To identify the surety of the output it is necessary to complete detailed simulation.

\section{SIMULATION \& EVALUATION}

\subsection{Simulation Environment}

The simulation model used in this paper is based on network simulator-2, which is used for protocol evaluation in experiment. The instructions of simulator can be utilize to specify the topology of the network and to specify the mode of node motion, also to configure the source of service and the receiver, to generate the data trace file [11].

Ns-2 provides good support for network simulation of routing and also multicast routing protocols for wired and wireless networks. NS2 is combination of two simulation tools which are the network simulator (ns) which contains various commonly used IP protocols and the network animator (nam) tool is utilize to actually visualize the network simulations.

\subsection{Traffic Model}

In this work we have used CBR continuous bit rate traffic sources for network simulation. In the pair of network made by source and destination nodes commonly distributed as randomly and also 512-byte data packets is used by us for analysis of task in experiment. The pairs count made for source and destination and also the sending rate of packet of node pairs are time to time changed by us to varying the load proposed for the network

\subsection{Mobility Model}

The mobility model [1][10] uses by us is the random waypoint model in a specified area of rectangular field which is configurations as 500 multiply be 500 area with the 100 nodes. In network communication every data packet starts its route from a specific random source location to a random node destination by a specified and randomly communication network speed. When it is reached to destination, then again another random node destination is specified. In the network simulation time is varied which responsible to effects the relative speeds of the nodes in network. Node mobility in network is same and traffic scenarios which are used by the protocols to get good output of experiments.

\subsection{Simulation Model}

Network Simulator NS2 [7], is an effective and simple event driven simulation software which is useful in testing of the implementation of logically networks and also make shorten the burden of actual network implementation. NS2 provides various capabilities to effective simulation of wired and wireless network and also support most of protocols. We have perform a simulation to evaluate the performance of Mobile Ad Hoc Network routing protocols ZRP based network throughput with various parameters[10][11].

Radio Model - Two Way Ground
Traffic Source -CBR

Packet Size - 512 Bytes

Network Speed - $10 \mathrm{~m} / \mathrm{s}$

Area - 500X500

Number of Nodes 100

MAC - Mac/802_11

Simulation Time $20,40,60,80 \& 100$

\subsection{Simulation Results}

To achieve our goal and aim we analyze behavior of ZRP protocol when load of nodes increases with mobility of nodes. We utilize network simulator 2.33 for this work. We conduct experiment on network of 100 nodes with and without mobility and ZRP protocol in different simulation times. After running tcl scripts in ns 2.33 simulator it creates trace files, we analyze these trace files by awk scripts and calculate some performance matrices as shown in table 1 and table 2 three types of matrices results taken on different simulation time.

Table 1.Experiment results in network of static nodes

\begin{tabular}{|c|c|c|c|}
\hline \multicolumn{4}{|c|}{ Network with static nodes using ZRP } \\
\hline $\begin{array}{c}\text { Simulation } \\
\text { Time }\end{array}$ & $\begin{array}{c}\text { Packet } \\
\text { Delivery } \\
\text { Ratio }\end{array}$ & $\begin{array}{c}\text { Average } \\
\text { End-to- } \\
\text { End Delay }\end{array}$ & $\begin{array}{c}\text { Average } \\
\text { Throughput }\end{array}$ \\
\hline 20 & 60.808 & 596.062 & 1885.65 \\
\hline 40 & 64.9717 & 593.162 & 1968.14 \\
\hline 60 & 65.7315 & 605.726 & 1969.36 \\
\hline 80 & 66.2021 & 627.995 & 1965.12 \\
\hline 100 & 66.6812 & 612.819 & 1964.44 \\
\hline
\end{tabular}

Table 2.Experiment results in network of random nodes

\begin{tabular}{|c|c|c|c|}
\hline \multicolumn{4}{|c|}{ Network with random nodes using ZRP } \\
\hline $\begin{array}{c}\text { Simulation } \\
\text { Time }\end{array}$ & $\begin{array}{c}\text { Packet } \\
\text { Delivery } \\
\text { Ratio }\end{array}$ & $\begin{array}{c}\text { Average } \\
\text { End-to- } \\
\text { End Delay }\end{array}$ & $\begin{array}{c}\text { Average } \\
\text { Throughput }\end{array}$ \\
\hline 20 & 58.3108 & 482.043 & 1728.62 \\
\hline 40 & 49.5302 & 581.523 & 1305.07 \\
\hline 60 & 38.1733 & 585.521 & 972.38 \\
\hline 80 & 31.1417 & 613.181 & 730.21 \\
\hline 100 & 26.2684 & 624.543 & 730.21 \\
\hline
\end{tabular}

\section{PERFORMANCE MATRICES}

Some important matrices we have evaluated for the analysis of routing protocol in different network scenario and different experimental setups in popular network simulator software [11].

\subsection{Average Throughput}

The average throughput is performance metrics which is a ratio of the total amount of data reaches to a specific receiver node from a sender node to the time which it takes. In the specific communication networks the network throughput is the average rate of a perfect message delivery in a particular transmission channel in simulated network. This data may be passed on a particular physical link or can be the logical link, 
or it can be passing through the specific particular network node in simulated network. The network throughput is generally calculated in the form of bits per second.

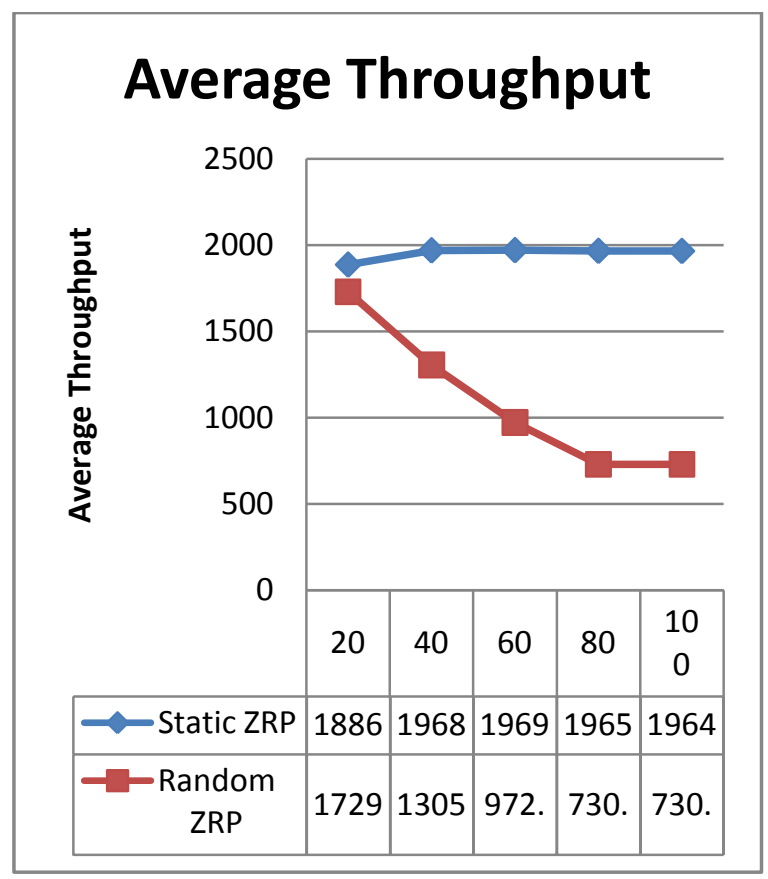

Fig. 2. Average Throughput

Based on simulation results, the throughput value of network based on static vector nodes and ZRP based network in different simulation time initially higher as 1885.65 but it increases as simulation time increases as on 100 simulation time it reach on 1964.44 whereas, the throughput value of network based on mobile random nodes and ZRP based network in different simulation time initially 1728.62 but it decrease constantly as simulation time increase as on 100 simulation time it reach on 730.21 which shows that in ZRP based network can provide good network throughput in static nodes features.

\subsection{Packet Delivery Ratio}

In network the packet delivery ratio is the ratio between lot of received packets by the destination node and the number of the data packets sent by the source node in network. It is the ratio between the number of packets actually transmitted by a network traffic source and the number of packets received by a network traffic sink. Packet delivery ratio can measures the loss rate as seen by transport protocols and as like, it characterizes the correctness and the efficiency of ad hoc network routing protocols. The high packet delivering ratio is very much desirable in any of the network. The packet ratio of the originated applications of the data packets of each of the protocol is able to deliver at varying time in network. Packet delivery ratio is the ratio of the number of delivered data packet to the destination. It illustrates level of the delivered data to the destination node in network.

$\sum$ Number of packet receive / $\sum$ Number of packet send

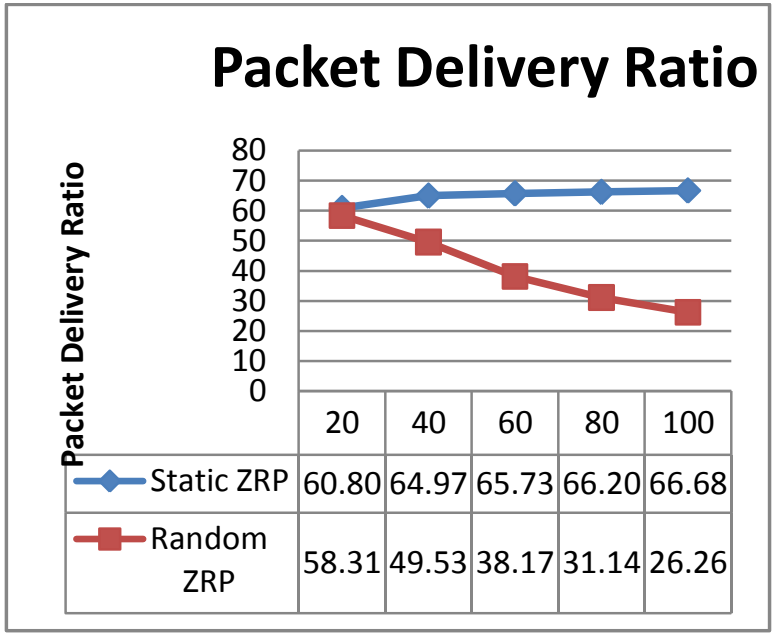

Fig. 3. Packet Delivery Ratio

Based on simulation results, the packet delivery ratio of network based on static vector nodes and ZRP based network in different simulation time initially low as 60.808 but it increases as simulation time increases as on 100 simulation time it reach on 66.6812 whereas, the packet delivery ratio of network based on mobile random nodes and ZRP based network in different simulation time initially 58.3108 , but it decrease as simulation time increase as on 100 simulation time and reach on 26.2684. Hence ZRP based network can provide good packet delivery ratio in static random nodes based network.

\subsection{Average End-to-End delay}

Average End to End Delay is the time which is elapsed when a data packet is sent from the source node in network and also it is received by the desired destination node in network. It includes delays as delay for route discovery, propagation time, time to transfer data, and intermediate queuing delays. It is the average time taken by a data packet to travel from source to destination. Also it includes the delay caused by the network route discovery process and the queue in data packet transmission from sender to receiver. Only the data packets which are successfully received to destinations will be counted.

$\sum$ (arrive time - send time) / $\sum$ Number of connections

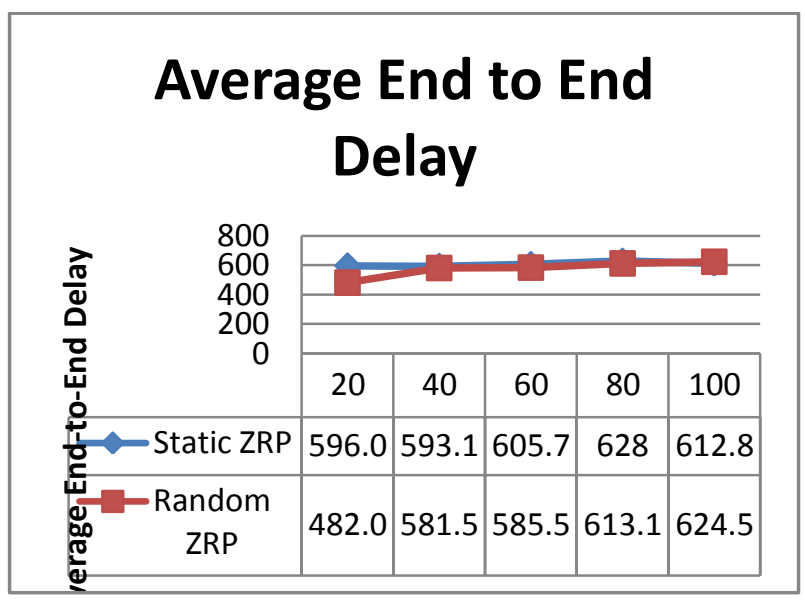

Fig. 4. Average End to End Delay 
Based on simulation results, the average end to end delay of network based on static vector nodes and ZRP based network in different simulation time initially low as 596.062 but it increases as simulation time increases as on 100 simulation time it reach on 612.819 whereas, the end to end delay of network based on mobile random nodes and ZRP protocol based network in different simulation time initially 482.043 , but it increase as simulation time increase as on 100 simulation time and reach on 624.543. It shows that ZRP based network can provide good end to end delay in static nodes based network.

\section{CONCLUSION}

In this paper we have simulate different types of networks with nodes having mobility or static features, and evaluate ZRP protocol for different matrices which shows network performances. According to results it can conclude that ZRP protocol provide good throughput and packet delivery ratio with static nodes in network, whereas node mobility in network effects on throughput in network and packet delivery ratio. Mobility of random nodes makes its performance low due to varying node position. Network with static node and ZRP provide initially low values for average end to end delay, but it increase with increase of simulation time.

\section{FUTURE WORK}

In the future, we like to perform extensive complex simulations to gain a more in-depth analysis of the wireless mobile ad hoc routing protocols. Also try to enhance some new feature in protocol. We like to introduce new protocol, not only for the various routing protocols but for more and more vast areas of wireless networking and various important concepts in computer network and communications.

\section{ACKNOWLEDGMENT}

My thanks to the experts who helped us to setup simulation environment so that we can experiment and analyze different scenarios and conclude better results.

\section{REFERENCES}

[1] $\mathrm{Xi} \mathrm{Hu} \mathrm{,} \mathrm{Jinkuan} \mathrm{Wang,} \mathrm{Cuirong} \mathrm{Wang,} \mathrm{"Mobility}$ adaptive Routing for Stable Transmission in Mobile Ad Hoc Networks " JOURNAL OF COMMUNICATIONS, VOL. 6, NO. 1, February 2011.

[2] Rakesh Poonia, Amit Kumar Sanghi, Dr. Dharm Singh "DSR Routing Protocol in Wireless Ad-hoc Networks: Drop Analysis" International Journal of Computer Applications (0975 - 8887) Volume 14- No.7, February 2011.

[3] Asma Ahmed, A. Hanan, Shukor A. R., Izzeldin M. "Routing in Mobile Ad hoc Network" IJCSNS International Journal of Computer Science and Network Security, VOL.11 No.8, August 2011.

[4] Beigh Bilal Maqbool Prof.M.A.Peer "Classification of Current Routing Protocols for Ad Hoc Networks - A
Review" International Journal of Computer Applications (0975 - 8887) Volume 7- No.8, October 2010.

[5] Sunil Taneja , Ashwani Kush "A Survey of Routing Protocols in Mobile Ad Hoc Networks" International Journal of Innovation, Management and Technology(2010 - 0248) Vol. 1, No. 3,August 2010.

[6] Md. Anisur Rahman, Md. Shohidul Islam, Alex Talevski "Performance Measurement of Various Routing Protocols in Ad-hoc Network" Proceedings of the International MultiConference of Engineers and Computer Scientists 2009 Vol I ,IMECS 2009, March 2009.

[7] Samyak Shah, Amit Khandre, Mahesh Shirole, Girish Bhole "Performance Evaluation of Ad Hoc Routing Protocols Using NS2 Simulation " Mobile and Pervasive Computing (CoMPC-2008).

[8] Padmini Misra, "Routing Protocols for Ad Hoc Mobile Wireless Networks" http://www.cis.ohio-state.edu/ jain/cis788-99/adhocrouting/index.html july2000.

[9] Furqan Haq and Thomas Kunz "Simulation vs. Emulation: Evaluating Mobile Ad Hoc Network Routing Protocols "Systems and Computer Engineering Carleton University Ottawa, Ont., Canada K1S 5B.

[10] Gianni A. Di Caro, "Analysis of simulation environments for mobile ad hoc networks" Technical Report No. IDSIA-24-03 IDSIA / USI-SUPSI , Dalle Molle Institute for Artificial Intelligence Galleria, Switzerland , December 2003.

[11] Karthik sadasivam, "Tutorial for Simulation-based Performance Analysis of MANET Routing Protocols in ns-2“, 2003.

[12] KAPANG LEGO , PRANAV KUMAR SINGH, DIPANKAR SUTRADHAR "Comparative Study of Adhoc Routing Protocol AODV, DSR and DSDV in Mobile Adhoc Network" Indian Journal of Computer Science and Engineering Vol.1 No.4 364-371.

[13] Gayatri Kapil IIMT, “AUGMENTED ZRP AS A ROUTING PROTOCOL FOR MANET", International Journal of Computational Engineering Research Vol, 03 Issue, 6 .

[14] Marc R. Pearlman, Student Member, IEEE, and Zygmunt J. Haas, Senior Member, "Determining the optimal configuration for the zone routing protocol" IEEE, IEEE JOURNAL ON SELECTED AREAS IN COMMUNICATIONS, VOL. 17, NO. 8, AUGUST 1999.

[15] Shaveta, Er. Navdeep Kumar, "Energy Saving in ZRP with Anycast Routing using NS2", INTERNATIONAL JOURNAL FOR ADVANCE RESEARCH IN ENGINEERING AND TECHNOLOGY, Volume 2, Issue VIII Aug 2014. 